\title{
An Empirical Study on the Characteristics of Question-Answering Process on Developer Forums
}

\author{
Yi Li ${ }^{1}$, Shaohua Wang ${ }^{1}$, Tien N. Nguyen ${ }^{2}$, Son Van Nguyen ${ }^{2}$, Xinyue $\mathrm{Ye}^{1}$, Yan Wang ${ }^{3}$ \\ ${ }^{1}$ Department of Informatics, New Jersey Institute of Technology, Newark, United States \\ $\{$ yl622, shaohua.wang, xinyue.ye $\} @$ njit.edu \\ ${ }^{2}$ Computer Science Department, The University of Texas at Dallas, United States, \{tien.n.nguyen,sonnguyen\}@utdallas.edu \\ ${ }^{3}$ Central University of Finance and Economics, China, dayanking@gmail.com
}

\begin{abstract}
Developer forums are one of the most popular and useful Q\&A websites on API usages. The analysis of API forums can be a critical resource for the automated question and answer approaches. In this paper, we empirically study three API forums including Twitter, eBay, and AdWords, to investigate the characteristics of question-answering process. We observe that $+60 \%$ of the posts on all three forums were answered by providing API method names or documentation. $+85 \%$ of the questions were answered by API development teams and the answers from API development teams drew fewer follow-up questions. Our results provide empirical evidences for us in a future work to build automated solutions to answer developer questions on API forums.
\end{abstract}

Index Terms-Question answering, API documentation

\section{INTRODUCTION}

Recently, developer question and answering (Q\&A) websites have become popular, critical and essential on-line resources that developers use to seek for their solutions on API usages, to share and learn knowledge of using APIs, and even to make discussions on the design of APIs [1].

Recently, two main types of developer Q\&A websites (DQA) have become popular. The first type is the generalpurpose Q\&A websites, for example, Stack Overflow [2]), taking any questions relevant to any APIs. The second type of DQA is the API Q\&A forums maintained by the libraries' providers, e.g., Twitter [3], and they accept only the questions relevant to the APIs of specific libraries. The main differences between the two DQAs can be summarized as follows: (1) Typically, an API forum is run by a library's provider and has the members from the development team to answer the questions relevant to the APIs of the libraries. Developers tend to ask API-specific questions on API forums. However, StackOverflow tends to deem the valid questions yet specific to a particular library as off-topic questions [4]. The API development teams on API forums can offer fast and right-to-the-point responses to the API specific questions [5]; and (2) Typically, the general-purpose DQA provides incentivesto improve the credibility of the responders and their public answers [6]. A question or an answer can be modified multiple times on SO, while the API Q\&A forums often do not allow developers to modify others' questions or answers. Due to those major differences, it is necessary to help API development teams answer more questions, and provide high-quality and right-tothe-point answers on API forums.

Extensive research [7], [8], [9], [10] has been devoted to studying StackOverflow (SO), one of the most popular DQA websites. However, despite the importance of API forums, little research has been focused on library-specific forums. In this paper, we set out to investigate the process of questionanswering on such forums. We empirically studied three popular API Q\&A forums, Twitter [3], eBay [11], and Google AdWords [12], to answer the following research questions:

RQ1. How are the questions answered?

In this RQ, we want to study how a question is answered by developers on an API forum. Our results indicate that majority of the questions were answered with provided API method names (or sometimes links to API documentation).

\section{RQ2. Who answer the questions?}

Similar to the general purpose DQA websites, any developer can answer a question on API forums. However, we observe that the majority of the studied questions were answered by API development teams.

\section{RQ3. What is the quality of answers?}

Our further analysis of the answered questions on API forums shows that the answers from API development teams have drawn fewer follow-up questions than the ones answered by other developers.

\section{EMPiRICAL STUdy Design}

Our overall goal is to understand the process of questionanswering on the library-specific forums.

Data Collection and Processing. We conducted an empirical study on three popular web-based Q\&A forums, including Twitter, eBay, and AdWords, to investigate the basics of developer API Q\&A forums and to motivate our study using our findings. We built a tool to crawl all of the questions and their answers from each of the aforementioned developer Q\&A forums (last access in April 2018). Table 1 shows that over $50 \%$ of the questions on each forum were not answered. Analysis Approach for RQs. We further analyze the answered questions of each forum to study who answer questions and how questions were answered. Using the confidence level 95\% with an interval 5\%, we randomly selected $368,358,374$ 
Table I: Statistics of Each library-specific Q\&A Forum.

\begin{tabular}{l|ccc}
\hline & Twitter & eBay & AdWords \\
\hline Total \# of questions & 16,874 & 6,204 & 23,731 \\
\# of questions with answers & 8,910 & 3,524 & 12,364 \\
\# of questions without answers & 7,964 & 2,680 & 11,367 \\
$\%$ of questions without answers & $52.8 \%$ & $56.8 \%$ & $52.1 \%$ \\
\hline
\end{tabular}

questions from 8,910, 5,231, and 14,245 questions having answers on Twitter, eBay, and AdWords, respectively. For each selected question, we manually studied the question and its answers to classify the question based on how it was answered.

There can be many metrics for measuring answer quality. For simplicity, in this short paper, we use the number of follow-up questions on an answer as one indicator to evaluate the quality of an answer in the analysis of RQ3.

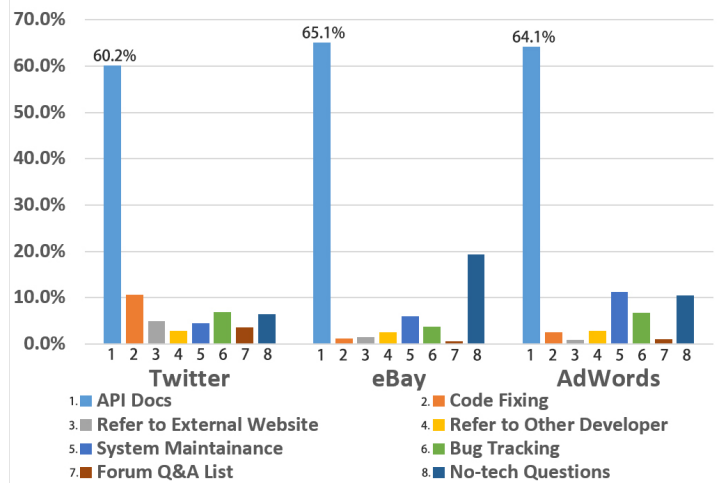

Figure 1: Eight Categories of Questions.

Results of RQs. Let us present the results of our study.

(RQ1.) The majority of the questions were answered with providing API method names (or sometimes links to API web pages). Figure 1 shows that we identify 8 categories of questions based on how they were answered. For example, the API Docs indicates the percentage of the studied questions were answered using an API document, and on average, 63\% of the questions were answered by using API documentation. The Code Fixing indicates the percentage of the questions that were for code errors, and not relevant to the API usages. The Refer to External Website refers to the percentage of the questions that can not be answered by API documents and need the information from other external websites.

(RQ2.) The majority of the studied questions were answered by API development teams. Figure $2 \mathrm{a}$ shows that $84-87.7 \%$ of the questions were answered by API development teams on the three forums, while other developers only answered a small portion of the questions.

(RQ3.) The answers from API development teams have drawn fewer follow-up questions than the ones answered by other developers. For example, Figure $2 b$ shows that only about $13 \%$ of the questions answered by the Twitter API development team drew follow-up questions, while about $65 \%$ of the questions answered by other developers do so. The other API forums share the same phenomenon.

\section{DISCUSSION}

Our three preliminary RQs show that it is important to assist API development teams to answer developer questions,

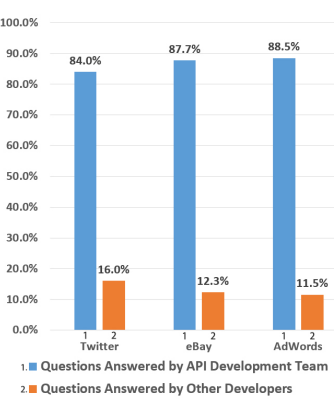

(a)

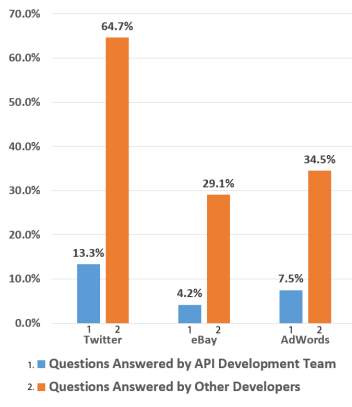

(b)
Figure 2: (a) Comparison between the Percentage of Questions Answered by API Development Team and Other Developers. (b) Comparison between the Percentage of Questions Answered by API Development Team and Other Developers, Receiving Follow-up Questions.

as on average, $+85 \%$ of the questions were answered by the API development team and fewer follow-up questions were triggered up after an answer was provided by an API development team member. Furthermore, on average, $+60 \%$ of the questions were answered using API document links or direct API method names. Thus, recommending relevant API documents to answer a question can be very useful.

\section{Threats TO VALIDITY}

Manual Analysis of API forum posts. During the labeling process, most answers can clearly show the relevant APIs. However, some answers can contain outdated links for API documents, which makes it very difficult to determine the relevant APIs. We discarded such answers to try our best to minimize the bias. Our process might bring bias to our results since we are not familiar with the APIs or from the development teams.

Selection of API forums. There are many API forums for different websites. In our research, we only focused on three very popular API forums, Twitter, eBay, and AdWords. Thus, we cannot claim that the result is general for all API forums.

\section{RELATED WORK}

There has been extensive research devoted to analyzing Stack Overflow, for example, such as analyzing obsolete answers [7], proposing guidelines for writing questions [8], discussing best-answer prediction models [9], learning to answer SO questions [10].

\section{CONCLUSION}

Our empirical results show that it is necessary to build automated solutions to help the API development teams to answer developers' questions. We plan to conduct further analysis on API forum posts and eventually propose solutions to automatically answer developer questions on API forums.

\section{ACKNOWLEDGMENTS}

This work was supported in part by the US National Science Foundation (NSF) grants CCF-1723215, CCF-1723432, TWC1723198, CCF-1518897, and CNS-1513263. 


\section{REFERENCES}

[1] L. Mamykina, B. Manoim, M. Mittal, G. Hripcsak, and B. Hartmann, "Design lessons from the fastest q\&a site in the west," in Proceedings of the SIGCHI conference on Human factors in computing systems. ACM, 2011, pp. 2857-2866.

[2] StackExchangeNetwork. Stack overflow. [Online]. Available: https: //stackoverflow.com/

[3] T. Inc. Twitter. [Online]. Available: https://twitter.com//

[4] M. Squire, "' should we move to stack overflow?" measuring the utility of social media for developer support," in 2015 IEEE/ACM 37th IEEE International Conference on Software Engineering, vol. 2. IEEE, 2015, pp. 219-228.

[5] P. K. Venkatesh, S. Wang, F. Zhang, Y. Zou, and A. E. Hassan, "What do client developers concern when using web apis? an empirical study on developer forums and stack overflow," in 2016 IEEE International Conference on Web Services (ICWS). IEEE, 2016, pp. 131-138.
[6] S. Wang, T.-H. P. Chen, and A. E. Hassan, "How do users revise answers on technical q\&a websites? a case study on stack overflow," IEEE Transactions on Software Engineering, 2018.

[7] H. Zhang, S. Wang, T.-H. P. Chen, Y. Zou, and A. E. Hassan, "An empirical study of obsolete answers on stack overflow," IEEE Transactions on Software Engineering, 2019.

[8] F. Calefato, F. Lanubile, and N. Novielli, "How to ask for technical help? evidence-based guidelines for writing questions on stack overflow," Information and Software Technology, vol. 94, pp. 186-207, 2018.

[9] —, "An empirical assessment of best-answer prediction models in technical q\&a sites," Empirical Software Engineering, vol. 24, no. 2, pp. 854-901, 2019.

[10] J. Li, A. Sun, and Z. Xing, "Learning to answer programming questions with software documentation through social context embedding," Information Sciences, vol. 448, pp. 36-52, 2018.

[11] eBay Inc. ebay. [Online]. Available: https://www.ebay.com/

[12] G. Inc. Adwords. [Online]. Available: https://ads.google.com/ 